\title{
Sensibilidad in vitro a quimioterapia como posible herramienta de medicina personalizada en pacientes con cáncer y ascitis maligna. Iniciativa del Centro Médico ABC
}

\author{
In vitro chemotherapy sensitivity as a potential \\ personalized medicine tool in cancer patients with \\ malignant ascites. An ABC Medical Center Iniciative
}

Alejandro Noguez Ramos, ${ }^{*}$ Daniela Shveid Gerson, ${ }^{*}$ Diana Alejandra Villegas Osorno, ${ }^{*}$ Alberto Villalobos Prieto, ${ }^{*}$ Juan Alberto Serrano Olvera, ${ }^{*}$ Guillermo Manuel Olivares Beltrán, ${ }^{*}$ Jesús Miguel Lázaro León, ${ }^{*}$ Gabriela Olivia Regalado Porras, ${ }^{*}$ Christian Patricio Camacho Limas, ${ }^{*}$ Eric Alejandro García López,,$\S$ Laura Daniela Campos Alvarado," Héctor Murrieta González," Alejandro Zentella Dehesa,,,,$++\neq \neq$ Raquel Gerson Cwilich $\$ \S$

Citar como: Noguez RA, Shveid GD, Villegas ODA, Villalobos PA, Serrano OJA, Olivares BGM et al. Sensibilidad in vitro a quimioterapia como posible herramienta de medicina personalizada en pacientes con cáncer y ascitis maligna. Iniciativa del Centro Médico ABC. An Med ABC. 2021; 66 (4): 238-248. https://dx.doi.org/10.35366/102923

\section{RESUMEN}

Introducción: Es imperativo desarrollar herramientas para elegir quimioterapia en pacientes con cáncer metastásico previamente tratados con varias líneas de quimoterapia. Estudios de sensibilidad a quimioterapia dilatan tiempo valioso para un paciente en este escenario, efectuarlo a partir de ascitis reduce considerablemente el tiempo necesario.

\section{ABSTRACT}

Introduction: In patients with metastatic cancer, it is imperative to develop tools for choosing chemotherapy. Chemotherapy sensitivity studies take months, doing it from ascites fluid considerably reduces the needed time. Objective: To evaluate the chemotherapy sensitivity in primary cultures of malignant cells obtained from ascites
* Departamento de Oncología Médica. Centro Médico ABC, IAP.

‡ Unidad de Bioquímica. Instituto Nacional de Ciencias Médicas y Nutrición «Salvador Zubirán».

$\S$ Departamento Sistemas Biológicos, Universidad Autónoma Metropolitana, Campus Xochimilco.

ๆ Tesista de licenciatura. Facultad de Ciencias, Universidad Nacional Autónoma de México.

\| Jefe del Departamento de Tomografía. División de Radiología e Imagen Molecular. Centro Médico ABC, IAP.

* Centro de Investigación. Centro Médico ABC, IAP.

执 Departamento de Medicina Genómica y Toxicología Ambiental. Instituto de Investigaciones Biomédicas, Universidad Nacional Autónoma de México.

$\S \S$ Directora del Centro de Cáncer, Departamento de Oncología Médica. Centro Médico ABC, IAP.

Ciudad de México, México.

Correspondencia:

Dra. Raquel Gerson Cwilich

E-mail: rgerson1@yahoo.com

Recibido: 26/05/2021. Aceptado: 11/11/2021. 
Objetivo: En cultivos primarios de células malignas obtenidas de ascitis evaluar la sensibilidad de quimioterapia para generar una herramienta de medicina personalizada. Material y métodos: Ensayo clínico en pacientes con cáncer metastásico y ascitis maligna del Centro Médico ABC. A partir de ascitis se obtuvieron cultivos celulares de cada paciente y fueron expuestos a diferentes quimioterapias, evaluamos la sensibilidad de acuerdo con el porcentaje de muerte celular. La concordancia entre la progresión a quimioterapia y su resistencia in vitro se reportó como total, parcial o nula. Resultados: Se incluyeron 24 pacientes. La mayoría mujeres 13 (54.1\%), la edad media fue $61 \pm 16.1$ años, $75 \%$ tenían ECOG 1-2, la mediana de líneas de tratamiento fue 2(RIC 1-4). Los tumores primarios fueron páncreas siete $(29.1 \%)$, mama seis (25\%), colorrectal cinco (20.8\%), hepatocelular tres $(12.5 \%)$, colangiocarcinoma dos $(8.3 \%)$ y peritoneo, uno $(4.1 \%)$. En 20 muestras $(80 \%)$ se logró establecer el cultivo celular. La concordancia parcial o total se encontró en 11 pacientes (55\%), por tumor primario fue de $100 \%$ en peritoneo, $83 \%$ en páncreas, $60 \%$ en colorrectal, $50 \%$ en colangiocarcinoma, $25 \%$ mama y $0 \%$ en hepatocelular. Conclusiones: Cultivar células neoplásicas a partir de ascitis y exponerlas a quimioterapia es factible y se realiza en dos semanas. La concordancia entre la resistencia a quimioterapia in vitro y la progresión clínica fue notoria en cáncer de peritoneo, páncreas y colorrectal.

Palabras clave: Cultivo celular, ascitis maligna, cáncer metastásico, medicina personalizada.

Nivel de evidencia: III

Abreviaturas:

QT = Quimioterapia

CMABC $=$ Centro Médico ABC.

$\mathrm{DE}=$ Desviación estándar

\section{INTRODUCCIÓN}

Se estima que la enfermedad metastásica es la causa de aproximadamente $90 \%$ de las muertes por cáncer. ${ }^{1}$ Con nuevos y mejores esquemas de tratamiento, la supervivencia de la mayoría de los pacientes con cáncer metastásico ha aumentado. En todos los tipos de cáncer evaluados como un conjunto se estimó una mejoría en la supervivencia a cinco años de aproximadamente $17 \%$. $^{2}$

Este aumento de supervivencia lleva consigo el incremento del número de pacientes candidatos a recibir más de dos líneas de tratamiento. Basados en ensayos clínicos aleatorizados fase 3 , las sociedades internacionales de oncología dan una pauta de tratamiento en las primeras líneas, ${ }^{3,4}$ con nivel de evidencia 1 . No obstante, en líneas subsecuentes la calidad de la información en la que se basan las recomendaciones disminuye considerablemente.

El escenario de un paciente con cáncer metastásico multitratado es cada vez más común y es todo un reto para el oncólogo médico. Es imperativo de- in order to generate a personalized medicine tool. Material and methods: Clinical trial in patients with metastatic cancer and malignant ascites at the $A B C$ Medical Center. Cell cultures were obtained from each patient and they were exposed to different chemotherapy's, according to the cell death percentage we evaluated the sensitivity. The concordance between progression to chemotherapy and its resistance in vitro was reported as: total, partial or null. Results: 24 patients were included. Most women 13 (54.1\%), the mean age was 61 \pm 16.1 years, $75 \%$ had ECOG 1-2, the lines treatment median was 2 (IQR 1-4). The primary tumors were pancreas 7 (29.1\%), breast $6(25 \%)$, colorectal $5(20.8 \%)$, hepatocellular $3(12.5 \%)$, cholangiocarcinoma 2 (8.3\%), and peritoneum 1(4.1\%). Cell culture was established in 20 samples (80\%). Partial or total concordance was found in 11 patients (55\%), according to primary tumor was $100 \%$ in peritoneum, $83 \%$ in pancreas, $60 \%$ in colorectal, $50 \%$ in cholangiocarcinoma, $25 \%$ in breast and $0 \%$ in hepatocellular. Conclusions: Culturing neoplastic cells from ascites and exposing them to chemotherapy is feasible and can be performed in two weeks. The concordance between in vitro chemotherapy resistance and clinical progression was notable in peritoneal, pancreatic, and colorectal cancer.

Keywords: Cell culture, malignant ascites, metastatic cancer, personalized medicine.

Level of evidence: III sarrollar herramientas que ayuden al clínico a tomar mejores decisiones.

La ascitis maligna es un signo de carcinomatosis peritoneal y representa aproximadamente $10 \%$ de todos los casos de ascitis. ${ }^{5}$ La etiología principal es el cáncer de ovario $(25-28 \%){ }^{6}$

Sin embargo, se puede observar en otro tipo de tumores como endometrio, trompa de Falopio, mama, colon, pulmón, páncreas o hígado. ${ }^{7}$ Incluso con tratamiento, la supervivencia global de los pacientes con ascitis maligna es mala, se estima una mediana de supervivencia de 5.6 meses. $^{8}$

La medicina personalizada es un término genérico sin una definición clara, y puede incorporar biomarcadores, pero también utiliza preferencias del paciente, factores sociales, factores económicos con el objetivo principal de seleccionar «el tratamiento adecuado para el paciente adecuado en el momento adecuado». ${ }^{9}$ La medicina que trata a cada paciente por igual debe terminar. Cada paciente, cada neoplasia, cada célula, cada gen son diferentes. Utilizar la quimiosensibilidad a la quimioterapia (QT) para dirigir el tratamiento en pacientes con cáncer metastásico ha generado éxitos reportados. ${ }^{10}$

Dirigir un tratamiento con herramientas que puedan predecir la sensibilidad o resistencia a los 
fármacos empleados no es una idea nueva. Los antibiogramas fueron descritos hace más de 70 años y se emplean cotidianamente para el tratamiento de infecciones bacterianas con tasas de éxito notables. ${ }^{11}$ Tecnologías con xenotrasplantes o generación de tumores en modelos murinos para evaluar la sensibilidad a QT han mostrado resultados prometedores. Sin embargo, estos abordajes no son baratos y el tiempo que requiere es de cuatro a seis meses. ${ }^{12,13}$ En pacientes con cáncer metastásico el tiempo es valioso, estos meses pueden ser toda su vida restante.

La capacidad de cultivar y caracterizar células recién aisladas de ascitis ofrece un importante sistema experimental que se asemeja más a la situación del paciente, proporcionando una mejor herramienta para desarrollar posibles intervenciones con mayor aplicabilidad terapéutica. ${ }^{14}$

Cultivar células neoplásicas suspendidas en líquido de ascitis es factible, reproducible, estandarizado, relativamente barato y rápido. Tener el número de células necesarias para realizar ensayos de sensibilidad a QT tomaría aproximadamente 10 días. ${ }^{15,16} \mathrm{El}$ tiempo necesario para obtener células tumorales de cada paciente a partir de líquido de ascitis es mucho menor en comparación con el necesario a partir de tejido sólido.

Con estos antecedentes planteamos estandarizar la técnica de cultivo de células malignas a partir de líquido de ascitis, exponer los cultivos a diferentes esquemas de agentes citotóxicos, evaluar la resistencia a QT in vitro y su concordancia con la exposición a QT previa en pacientes con cáncer metastásico con el objetivo de desarrollar una herramienta para la elección de QT.

Objetivo: deesarrollar una herramienta clínica para elegir un esquema de tratamiento personalizado para pacientes multitratados en un lapso breve; utilizando cultivos primarios de células neoplásicas obtenidas de líquido de ascitis de pacientes con cáncer, y evaluar la sensibilidad en cultivos celulares con diferentes esquemas de QT.

\section{MATERIAL Y MÉTODOS}

Se trata de un ensayo clínico realizado en el Centro Médico ABC (CMABC). El protocolo fue aprobado por nuestro centro con registro ABC 19-31. Previo consentimiento informado, del 21 de junio de 2019 al 28 de febrero de 2021 se incluyeron pacientes con cáncer metastásico y ascitis a los que se les realizó una paracentesis por motivos independientes a nuestro protocolo.

Las muestras fueron centrifugadas a $2,500 \mathrm{rpm}$ por cinco minutos para obtener un pellet celular. Se descartó el líquido sobrenadante. El pellet de células se suspendió en medio de cultivo y se tomó una alícuota para el conteo de células. Las células obtenidas de cada paciente se sembraron en medio de cultivo RPMI 1,640 , suplementado con $10 \%$ de suero fetal bovino. Se incubaron a $37{ }^{\circ} \mathrm{C}$ en una atmósfera de $5 \% \mathrm{CO}_{2}$ con $100 \%$ de humedad relativa. El cultivo celular se expandió durante 96 horas y la proliferación celular se monitoreaba a través de la aparición de figuras mitóticas. Las células se cosecharon y cuantificaron para generar cultivos en monocapa (2D) y esferoides (3D).

Las células para el cultivo en 3D en lugar de ser sembradas en placas Petri p-60, se sembraron $100 \mu \mathrm{L}$ de suspensión celular (aproximadamente 30,000 cel/ $\mathrm{cm}^{2}$ ) en la microplaca formadora de esferoides con una superficie de ultra baja adherencia. La microplaca se incubó a $37{ }^{\circ} \mathrm{C}$ a $5 \% \mathrm{CO}_{2}$ en $100 \%$ de humedad, durante 48 horas para la formación del esferoide. Cada tercer día se cambiaba el medio de cultivo suplementado hasta que las células alcanzaron confluencia y se realizó el antibiograma.

Los cultivos celulares fueron expuestos a diversos esquemas de QT. La concentración farmacológica empleada en los ensayos in vitro emula la concentración sérica en el paciente. Para cada fármaco se utilizaron tres dosis: $0.1 \mathrm{x}, 1.0 \mathrm{x}$ y $10 \mathrm{x}$ de la dosis farmacológica estándar indicada en la clínica. Después de 48 horas de exposición a los fármacos evaluamos la viabilidad de los cultivos celulares y se reportó el porcentaje de muerte celular.

$\mathrm{Al}$ final del experimento los cultivos 2D fueron teñidos con cristal violeta y los 3D con azul de tripano. Después los cultivos 2D fueron lavados con PBS para remover el excedente de colorante, tratados con ácido acético para recuperar el colorante absorbido por las células y este colorante fue leído en un espectrofotómetro. La absorbancia es proporcional al número de células sobrevivientes. Después de 50\% de muerte celular se reportó sensibilidad y menor al 50\% resistencia al esquema de QT evaluado.

Analizamos entonces la relación entre los patrones de resistencia celular in vitro y la evolución clínica previa de cada paciente.

La QT utilizada fue: cisplatino, carboplatino, oxaliplatino, paclitaxel, docetaxel, irinotecan, topotecan, doxorrubicina, gemcitabina y 5 -fluorouracilo.

\section{RESULTADOS}

Se identificaron 24 pacientes con cáncer metastásico y ascitis maligna, todos aceptaron participar en el protocolo. Un paciente participó en dos ocasiones en diferentes momentos de su tratamiento. 
Tabla 1: Características basales de la población. $\mathrm{N}=24$.

\begin{tabular}{ll}
\hline & $n(\%)$ \\
\hline Edad & $61 \pm 16.1$ \\
Hombres & $11(45.9)$ \\
Mujeres & $13(54.1)$ \\
Antecedentes heredofamiliares de cáncer & $12(50)$ \\
Antecedente de tabaquismo & $8(33.3)$ \\
ECOG & \\
1 & $9(37.5)$ \\
2 & $9(37.5)$ \\
3 & $3(12.5)$ \\
4 & $3(12.5)$ \\
Línea de tratamiento & $2(\mathrm{RIC} 2-4)$ \\
Número de drogas a las que progresó & $3(\mathrm{RIC} 1-5)$ \\
Tumor primario & \\
Páncreas & $7(29.1)$ \\
Mama & $6(25.0)$ \\
Colorrectal & $5(20.8)$ \\
Carcinoma hepatocelular & $3(12.5)$ \\
Colangiocarcinoma & $2(8.3)$ \\
Peritoneo & $1(4.1)$ \\
\hline
\end{tabular}

ECOG = Escala del Eastern Cooperative Oncology Group, RIC = Rango Intercuartil. (Libre distribución).
La mayoría de los pacientes fueron mujeres 13 (54.1\%), la media de edad fue de $61 \pm 16.1$ años, 50\% tenían antecedentes heredofamiliares de cáncer, $33 \%$ antecedente de tabaquismo, $75 \%$ de los pacientes tenían ECOG 1-2, la mediana de líneas de tratamiento fue de 2 (RIC 1-4). Los tumores primarios fueron páncreas siete $(29.1 \%)$, mama seis $(25 \%)$, colorrectal cinco $(20.8 \%)$, carcinoma hepatocelular tres $(12.5 \%)$, colangiocarcinoma dos $(8.3 \%)$ y peritoneo uno $(4.1 \%)$ (Tabla 1).

En 20 muestras (80\%) se logró establecer el cultivo celular, en cinco (20\%) no hubo crecimiento celular (Figuras 1 y 2). La tasa de obtención de cultivo celular fue de:

1. Colangiocarcinoma $100 \%(\mathrm{~N}=$ dos $)$

2. Peritoneo $100 \%(\mathrm{~N}=$ uno $)$

3. Páncreas $85.7 \%(\mathrm{~N}=$ siete $)$

4. Colorrectal $83 \%(\mathrm{~N}=$ cinco $)$

5. Carcinoma hepatocelular $75 \%(\mathrm{~N}=$ tres $)$

6. Mama $66 \%(\mathrm{~N}=$ seis $)$

Se realizaron 20 ensayos de sensibilidad a quimioterapia. La media de drogas probadas por muestra fue de $8 \pm 1.8$ (Figura 3).
La mediana de muerte celular por droga fue:

1. Cisplatino 47\% (significado RIC 38-73)

2. Carboplatino 9\% (RIC 5-16)

3. Oxaliplatino 27\% (RIC 19-34)

4. Paclitaxel 40\% (RIC 31-54)

5. Docetaxel 90\% (RIC 87-94)

6. Irinotecan $33 \%$ (RIC 22-41)

7. Topotecan $15 \%$ (RIC 21-37)

8. Doxorrubicina 49 (RIC 26-79)

9. Gemcitabina 56\% (RIC 49-62\%)

10.5-Fluorouracilo 29\% (RIC 22-44).

Los datos de muerte celular in vitro por agente de quimioterapia de todos los pacientes se muestran en la Tabla 2. Dado que algunos fármacos no eran relevantes para el tratamiento, no existen resultados para esos fármacos. Con fines de homogeneidad en la presentación de los resultados los fármacos no probados se identifican como NP (no probados) (Tabla 2).

La relación maniobra-resultado, es decir, la concordancia entre la progresión a citotóxicos previos y la resistencia a quimioterapia in vitro se analizaron por tumor primario. Definimos tres patrones de concordancia.

1. Concordancia total: las drogas a las que había progresado mostraban resistencia in vitro.

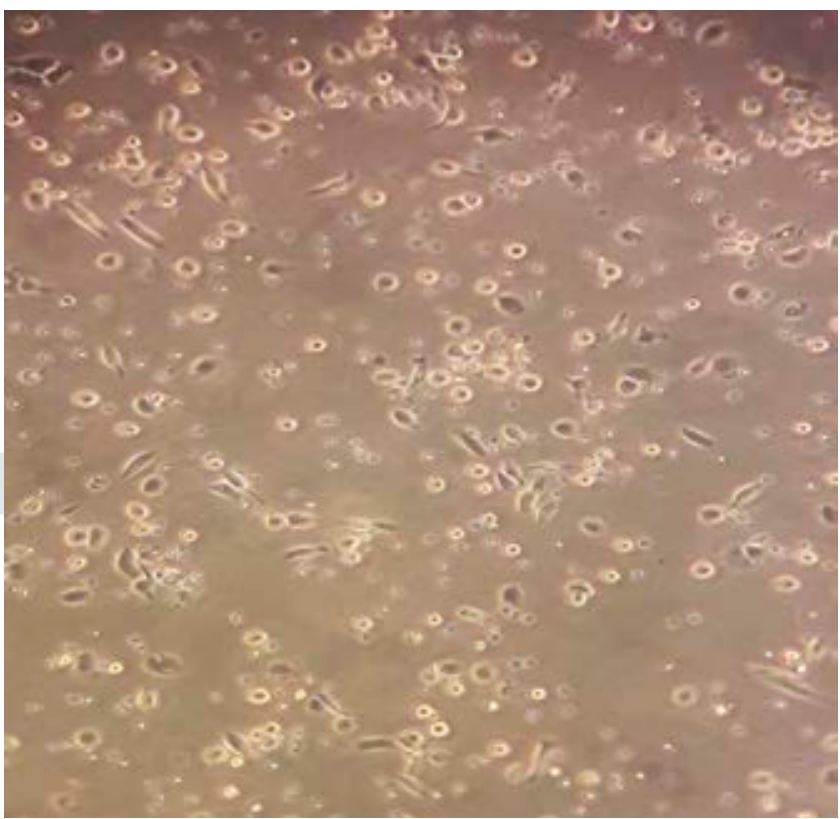

Figura 1: Células tumorales en cultivo celular vistas al microscopio. 
Figura 2:

Diagrama de flujo

QT = Quimioterapia, Sensibilidad in vitro a

$\mathrm{QT}=$ muerte celular $>50 \%$, Resistencia in vitro a $\mathrm{QT}=$ Muerte celular $<50 \%$.

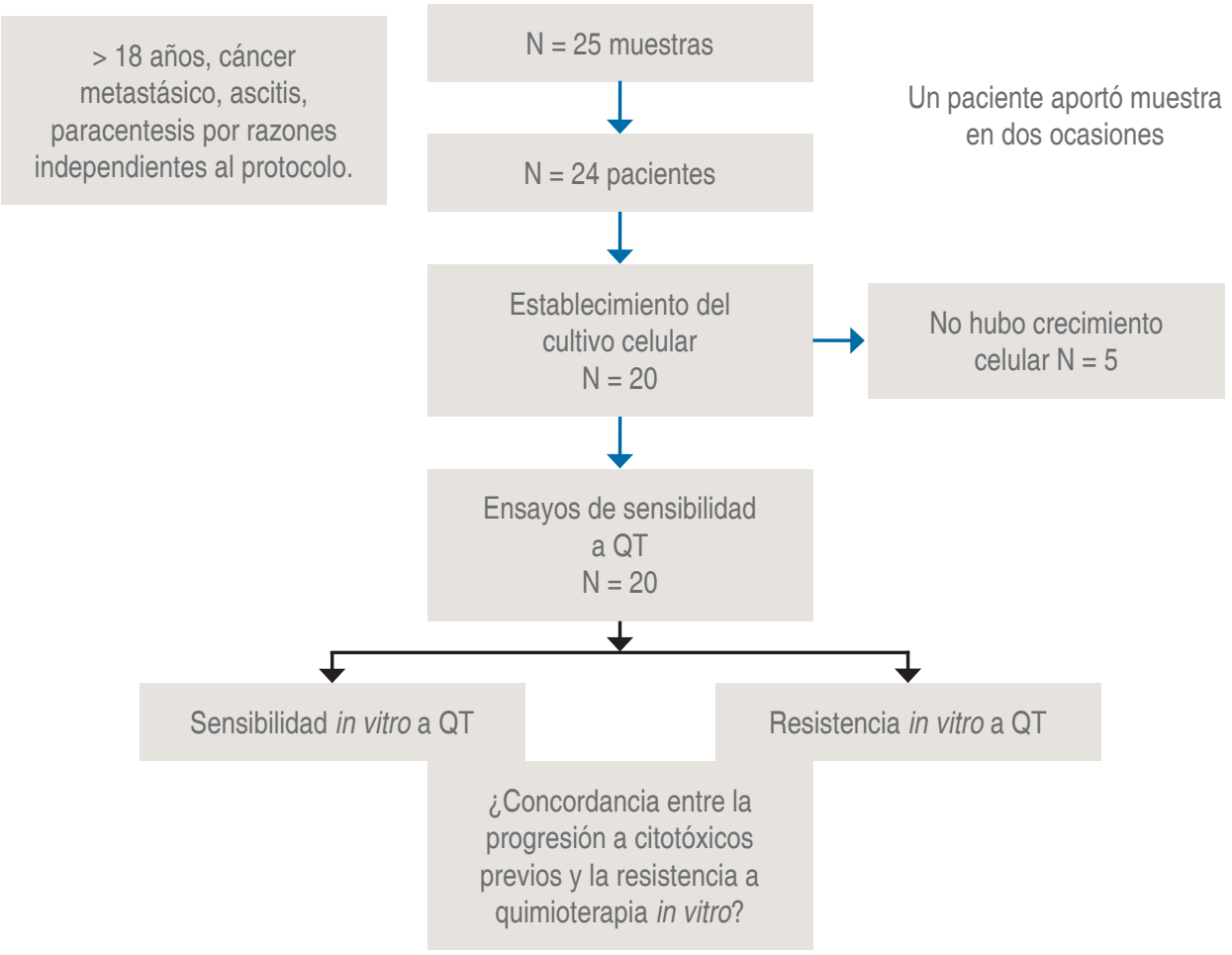

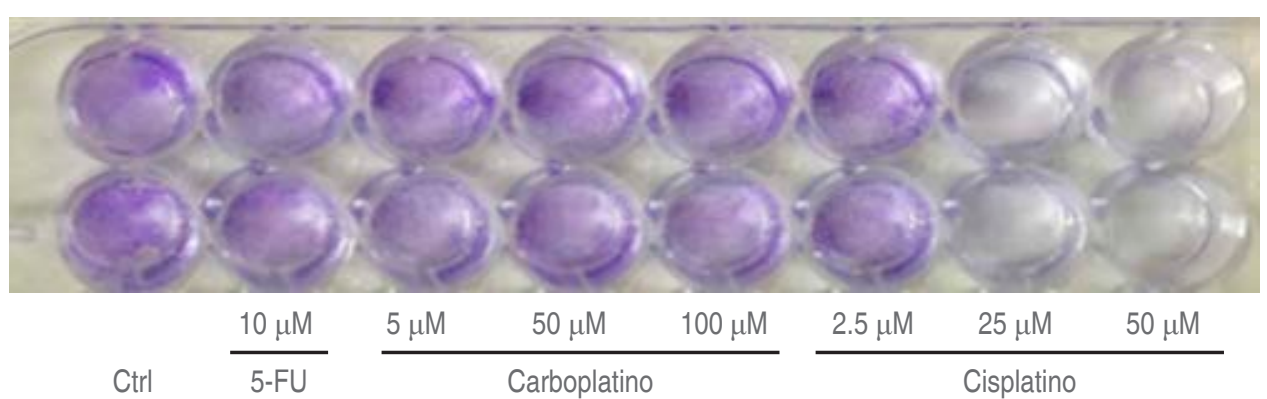

Valoración de viabilidad con cristal violeta, imagen representativa de los pozos teñidos al final de las 48 horas de la exposición a citotóxicos. Los pozos muestran por duplicado la tinción de un ensayo particular. La intensidad de la tinción es proporcional a la viabilidad celular. La cuantificación se hizo solubilizando el colorante en isopropanol/agua y leyendo su absorbancia en el espectrofotómetro. El porcentaje de muerte se calculó tomando como $100 \%$ los pozos sin células.
Figura 3:

Cultivo 2D.
2. Concordancia parcial: algunas de las drogas a las que había progresado mostraban resistencia in vitro.

3. Concordancia nula: no hay relación entre la progresión y la resistencia in vitro.

La concordancia parcial o total entre la resistencia a quimioterapia in vitro y la progresión clínica se encontró en 11 pacientes (55\%) y fue nula en nueve $(45 \%)$. La concordancia por tumor primario fue de $100 \%$ de peritoneo, $83 \%$ en cáncer de páncreas, $60 \%$ en cáncer colorrectal, $50 \%$ en colangiocarcinoma, $25 \%$ en cáncer de mama y $0 \%$ en carcinoma hepatocelular.

\section{Concordancia por tumor primario}

Se incluyeron dos pacientes con colangiocarcinoma, uno de ellos en cuarta línea de tratamiento, el cual muestra resistencia in vitro a las drogas a las que fue expuesto el paciente previamente y un paciente sin exposición a citotóxicos previos.

Se analizaron cinco pacientes con cáncer colorrectal metastásico, la mediana de líneas de tratamiento en este grupo fue de dos. Todos a partir de líquido de ascitis. Dos pacientes mostraron concordancia total entre la resistencia in vitro y la progre- 
Tabla 2: Muerte celular por tumor primario y tipo de quimioterapia en líquido de ascitis.

\begin{tabular}{|c|c|c|c|c|c|c|c|c|c|c|c|c|c|}
\hline Identificación & Primario & Línea & Cisplatino & Carboplatino & Oxilaplatino & Paclitaxel & Docetaxel & Irinotecan & Topotecan & Etopósido & Doxorrubicina & Gemcitabina & $5 \mathrm{FU}$ \\
\hline AAALC3 & Colangiocarcinoma & 4 & $71.6(2.9)$ & $\begin{array}{c}7.1(1.4) \\
\mathrm{R}\end{array}$ & $\begin{array}{c}28.5(3.0) \\
R\end{array}$ & $53.8(0.4)$ & $94.7(0.1)$ & $\begin{array}{c}20.3(3.4) \\
\mathrm{R}\end{array}$ & NP & NP & NP & $59.6(2.8)$ & $\begin{array}{c}24.1(3.6) \\
R\end{array}$ \\
\hline LUCALC4 & Colangiocarcinoma & 0 & $\begin{array}{c}45.6(1.6) \\
\mathrm{R}\end{array}$ & $\begin{array}{c}6.7(4.4) \\
\mathrm{R}\end{array}$ & $\begin{array}{c}35.8(4.5) \\
\mathrm{R}\end{array}$ & $\begin{array}{c}47.3(1.2) \\
\mathrm{R}\end{array}$ & $87.0(0.5)$ & $\begin{array}{c}31.5(3.2) \\
\mathrm{R}\end{array}$ & $\begin{array}{c}37.2(2.2) \\
\mathrm{R}\end{array}$ & NP & $\begin{array}{c}29.3(1.8) \\
R\end{array}$ & $\begin{array}{c}37.2(2.2) \\
\mathrm{R}\end{array}$ & $\begin{array}{c}29.3(1.8) \\
\mathrm{R}\end{array}$ \\
\hline LUCALC12 & Colorrectal & 3 & $\begin{array}{c}40.8(3.5) \\
R\end{array}$ & $\begin{array}{c}5.9(2.1) \\
\mathrm{R}\end{array}$ & $\begin{array}{c}19.4(0.9) \\
R\end{array}$ & $\begin{array}{c}36.6(2.2) \\
\mathrm{R}\end{array}$ & $83.6(0.4)$ & $\begin{array}{c}19.9(3.6) \\
\mathrm{R}\end{array}$ & $\begin{array}{c}12.7(9.3) \\
R\end{array}$ & NP & NP & $59.3(2.0)$ & $\begin{array}{c}21.3(2.0) \\
R\end{array}$ \\
\hline YDLC10 & Colorrectal & 2 & NP & $\begin{array}{c}20.5(4.6) \\
\mathrm{R}\end{array}$ & $\begin{array}{c}42.1(0.8) \\
\mathrm{R}\end{array}$ & $\begin{array}{c}25.9(3.2) \\
\mathrm{R}\end{array}$ & $97.7(0.7)$ & $\mathrm{NP}$ & $\begin{array}{c}27.7(3.2) \\
R\end{array}$ & NP & 64 & NP & $\begin{array}{c}31.8(0.8) \\
R\end{array}$ \\
\hline AAALC7 & Colorrectal & 0 & $65.0(1.4)$ & $\begin{array}{c}4.2(4.4) \\
\quad \mathrm{R}\end{array}$ & $\begin{array}{c}26.6(3.3) \\
\mathrm{R}\end{array}$ & $\begin{array}{c}50.1(4.7) \\
\mathrm{R}\end{array}$ & $90.3(1.7)$ & $\begin{array}{c}26.2(4.9) \\
\mathrm{R}\end{array}$ & NP & NP & NP & $56.8(3.8)$ & $\begin{array}{c}27.6(3.5) \\
R\end{array}$ \\
\hline AAALC10 & Colorrectal & 1 & $76.1(1.7)$ & $\begin{array}{c}5(4.3) \\
R\end{array}$ & $\begin{array}{c}18(2.8) \\
\mathrm{R}\end{array}$ & $\begin{array}{c}38.2(4.8) \\
\mathrm{R}\end{array}$ & $90.8(0.3)$ & $\begin{array}{c}29.8(3.6) \\
R\end{array}$ & NP & NP & NP & $56.6(0.1)$ & $\begin{array}{c}25.3(1.6) \\
R\end{array}$ \\
\hline YUSLC3 & Colorrectal & 2 & $\begin{array}{c}18.7(4.5) \\
\mathrm{R}\end{array}$ & $\begin{array}{c}8.3(2.5) \\
R\end{array}$ & $\begin{array}{c}19.4(3.7) \\
\mathrm{R}\end{array}$ & $\mathrm{NP}$ & $90.3(1.1)$ & $\begin{array}{c}21.8(3.8) \\
R\end{array}$ & $\begin{array}{c}16(2.9) \\
\mathrm{R}\end{array}$ & NP & NP & $\begin{array}{c}41.4(1.5) \\
\mathrm{R}\end{array}$ & $72.6(1.3)$ \\
\hline ONCOLC1 & C Hepatocelular & 2 & $77.8(0.7)$ & $\begin{array}{c}16.6(5.5) \\
R\end{array}$ & $\begin{array}{c}40.6(5.6) \\
R\end{array}$ & $\begin{array}{c}65.8(4.1) \\
R\end{array}$ & $94.6(0.5)$ & $\begin{array}{c}44.9(4.9) \\
\mathrm{R}\end{array}$ & NP & NP & NP & $65.3(4.6)$ & $\begin{array}{c}26.8(4.3) \\
R\end{array}$ \\
\hline AAALC5 & C Hepatocelular & 1 & $70.1(0.8)$ & $\begin{array}{c}10.0(2.2) \\
\mathrm{R}\end{array}$ & $\begin{array}{c}30.2(3.1) \\
R\end{array}$ & $62.1(0.4)$ & $94.9(0.02)$ & $\begin{array}{c}42.1(2.6) \\
\mathrm{R}\end{array}$ & NP & NP & NP & $\begin{array}{c}49.5(2.7) \\
\mathrm{R}\end{array}$ & $53.0(0.2)$ \\
\hline AAALC2 & Mama & 2 & $78.6(1.1)$ & $\begin{array}{c}17.0(3.6) \\
\mathrm{R}\end{array}$ & $\begin{array}{c}23.6(3.8) \\
R\end{array}$ & $\begin{array}{c}43.5(2.4) \\
\mathrm{R}\end{array}$ & $96.0(0.5)$ & $\begin{array}{c}38.7(3.8) \\
\mathrm{R}\end{array}$ & NP & NP & $96.6(0.4)$ & $67.7(3.6)$ & $\begin{array}{c}44.7(2.3) \\
\mathrm{R}\end{array}$ \\
\hline YDA705 & Mama & 2 & $\begin{array}{c}10(1.0) \\
\mathrm{R}\end{array}$ & NP & NP & $\begin{array}{c}14.0(1.2) \\
\mathrm{R}\end{array}$ & NP & NP & NP & NP & $72.0(2.0)$ & NP & $\begin{array}{c}8.0(1.1) \\
R\end{array}$ \\
\hline LUCALC13 & Mama & 3 & $69.5(2.3)$ & $\begin{array}{c}19.2(3.4) \\
R\end{array}$ & $\begin{array}{c}25.1(4.2) \\
\mathrm{R}\end{array}$ & $\begin{array}{c}36.2(6.8) \\
\mathrm{R}\end{array}$ & $87.8(0.2)$ & $\begin{array}{c}15.7(3.8) \\
\mathrm{R}\end{array}$ & $15.9(3.4)$ & NP & NP & NP & $\begin{array}{c}20(4.3) \\
R\end{array}$ \\
\hline AAALC13 & Mama & 2 & $\begin{array}{c}42.9(4.9) \\
\mathrm{R}\end{array}$ & $\begin{array}{c}2.1(4.2) \\
\quad \mathrm{R}\end{array}$ & $\begin{array}{c}30.4(6.1) \\
R\end{array}$ & $\begin{array}{c}40.5(3.2) \\
\mathrm{R}\end{array}$ & $94.0(0.2)$ & $\begin{array}{c}35.3(0.3) \\
\mathrm{R}\end{array}$ & NP & NP & NP & $57.0(1.5)$ & $\begin{array}{c}37.1(1.4) \\
R\end{array}$ \\
\hline AAALC4 & Páncreas & 1 & NP & NP & $\begin{array}{c}33.7(2.2) \\
\mathrm{R}\end{array}$ & NP & NP & $\begin{array}{c}34.7(0.9) \\
R\end{array}$ & NP & NP & NP & $\begin{array}{c}44.9(2.8) \\
\mathrm{R}\end{array}$ & $\begin{array}{c}33.9(3.0) \\
\mathrm{R}\end{array}$ \\
\hline YDLCAP8 & Páncreas & 2 & NP & NP & $\begin{array}{c}10.0(1.0) \\
R\end{array}$ & $\begin{array}{c}8.0(1.1) \\
\mathrm{R}\end{array}$ & NP & $\mathrm{NP}$ & NP & NP & NP & $62.0(2.0)$ & $\begin{array}{c}4.0(1.1) \\
\mathrm{R}\end{array}$ \\
\hline LUCALC14 & Páncreas & 3 & $\begin{array}{c}41(3.2) \\
\mathrm{R}\end{array}$ & $\begin{array}{c}9.8(0.4) \\
R\end{array}$ & $\begin{array}{c}19.2(1.9) \\
\mathrm{R}\end{array}$ & $\begin{array}{c}40.5(0.5) \\
\mathrm{R}\end{array}$ & $79.6(1.1)$ & $\begin{array}{c}24.6(3.2) \\
R\end{array}$ & $37.9(6.2)$ & $\mathrm{NP}$ & NP & NP & $\begin{array}{c}29.5(7.8) \\
\mathrm{R}\end{array}$ \\
\hline$A B C L C 1$ & Páncreas & 2 & $76.2(2.7)$ & $\begin{array}{c}11.9(3.7) \\
\mathrm{R}\end{array}$ & $\begin{array}{c}39.3(2.9) \\
R\end{array}$ & $\begin{array}{c}66.2(5.6) \\
\mathrm{R}\end{array}$ & $93.2(0.1)$ & $\begin{array}{c}38.9(1.9) \\
\mathrm{R}\end{array}$ & NP & NP & $\mathrm{NP}$ & $76.08(2.1)$ & $58.0(3.5)$ \\
\hline AAALC8 & Páncreas & 1 & $\begin{array}{c}36.3(3.6) \\
\mathrm{R}\end{array}$ & $\begin{array}{c}2.3(0.3) \\
\mathrm{R}\end{array}$ & $\begin{array}{c}27.0(4.5) \\
R\end{array}$ & $\begin{array}{c}32.9(3.9) \\
\mathrm{R}\end{array}$ & $89.8(0.3)$ & $\begin{array}{c}46.3(2.5) \\
R\end{array}$ & $\mathrm{NP}$ & NP & $\mathrm{NP}$ & $\begin{array}{c}50.8(1.7) \\
\mathrm{R}\end{array}$ & $\begin{array}{c}44.6(1.4) \\
\mathrm{R}\end{array}$ \\
\hline ABCLC6 & Páncreas & 2 & $\begin{array}{c}47.7(0.7) \\
\mathrm{R}\end{array}$ & $\begin{array}{c}18.5(0.1) \\
\mathrm{R}\end{array}$ & $\begin{array}{c}28.5(1.2) \\
R\end{array}$ & $57.0(0.2)$ & $90.1(0.3)$ & $\begin{array}{c}45.1(9.5) \\
\mathrm{R}\end{array}$ & $\mathrm{NP}$ & NP & $\mathrm{NP}$ & $55.3(0.5)$ & $\begin{array}{c}43.2(4.8) \\
\mathrm{R}\end{array}$ \\
\hline YDLCP4 & Páncreas & 5 & $\begin{array}{c}30.8(0.5) \\
R\end{array}$ & $\mathrm{NP}$ & $\mathrm{NP}$ & $\begin{array}{c}14.0(0.7) \\
R\end{array}$ & $\begin{array}{c}27.0(1.6) \\
R\end{array}$ & NP & $\mathrm{NP}$ & NP & $\begin{array}{c}26.0(2.0) \\
\mathrm{R}\end{array}$ & NP & $\begin{array}{c}9.0(0.2) \\
\mathrm{R}\end{array}$ \\
\hline
\end{tabular}

$\mathrm{R}=$ resistencia = muerte celular $<50 \%$ con la dosis farmacológica $1.0 \mathrm{X}, \mathrm{NP}=$ fármaco no probado, $5 \mathrm{FU}=5$ fluorouracilo. 
An Med ABC. 2021; 66 (4): 238-248

Tabla 3: Concordancia entre la progresión a citotóxicos previos y la resistencia a quimioterapia in vitro dependiendo del tumor primario.

\begin{tabular}{|c|c|c|c|c|c|c|}
\hline Identificación & Tumor primario & Línea & $\begin{array}{l}\text { Drogas a las que } \\
\text { progresó }\end{array}$ & Resistencia in vitro & Sensibilidad in vitro & Concordancia \\
\hline AAALC3 & Colangiocarcinoma & 4 & $\begin{array}{c}5 \text { FU } \\
\text { Irinotecan } \\
\text { Oxaliplatino } \\
\text { carboplatino } \\
\text { Gemcitabina } \\
\text { NAB Paclitaxel } \\
\text { Pemetrexed } \\
\text { Bevacizumab }\end{array}$ & $\begin{array}{l}5 \text { FU } \\
\text { Irinotecan } \\
\text { Oxaliplatino } \\
\text { Carboplatino }\end{array}$ & $\begin{array}{c}\text { Cisplatino } \\
\text { Paclitaxel } \\
\text { Docetaxel } \\
\text { Gemcitabina }\end{array}$ & Total \\
\hline LUCALC4 & Colangiocarcinoma & 0 & Ninguna & $\begin{array}{c}\text { Cisplatino } \\
\text { Carboplatino } \\
\text { Oxaliplatino } \\
\text { Paclitaxel } \\
\text { Irinotecan } \\
\text { Topotecan } \\
\text { Doxorrubicina } \\
\text { Gemcitabina } \\
5 \text { FU }\end{array}$ & Docetaxel & Nula \\
\hline LUCALC12 & Colorrectal & 3 & $\begin{array}{l}5 \mathrm{FU} \\
\text { Oxaliplatino } \\
\text { Irinotecan } \\
\text { Bevacizumab }\end{array}$ & $\begin{array}{c}5 \text { FU } \\
\text { Oxaliplatino } \\
\text { Irinotecan } \\
\text { Cisplatino } \\
\text { Carboplatino } \\
\text { Paclitaxel } \\
\text { Topotecan }\end{array}$ & $\begin{array}{l}\text { Docetaxel } \\
\text { Gemcitabina }\end{array}$ & Total \\
\hline YDLC10 & Colorrectal & 2 & $\begin{array}{l}\text { Oxaliplatino } \\
\text { Capecitabina }\end{array}$ & $\begin{array}{l}\text { Oxaliplatino } \\
\quad 5 \text { FU } \\
\text { Carboplatino } \\
\text { Paclitaxel } \\
\text { Topotecan }\end{array}$ & $\begin{array}{c}\text { Docetaxel } \\
\text { Doxorrubicina }\end{array}$ & Total \\
\hline AAALC7 & Colorrectal & 0 & Ninguna & $\begin{array}{c}\text { Carboplatino } \\
\text { Oxaliplatino (26\%) } \\
\text { Paclitaxel } \\
\text { Irinotecan } \\
5 \text { FU }(27 \%)\end{array}$ & $\begin{array}{c}\text { Cisplatino } \\
\text { Docetaxel } \\
\text { Gemcitabina }\end{array}$ & Nula \\
\hline AAALC10 & Colorrectal & 1 & $\begin{array}{l}\text { Ninguna } \\
\text { (dos ciclos de } \\
\text { FOLFOX) }\end{array}$ & $\begin{array}{c}\text { Carboplatino } \\
\text { Oxaliplatino (18\%) } \\
\text { Paclitaxel } \\
\text { Irinotecan } \\
5 \text { FU }(25 \%)\end{array}$ & $\begin{array}{c}\text { Cisplatino } \\
\text { Docetaxel } \\
\text { Gemcitabina }\end{array}$ & Nula \\
\hline YUSLC3 & Colorrectal & 2 & $\begin{array}{c}\text { Oxaliplatino } \\
\text { Irinotecan } \\
\text { Capecitabina } \\
5 \text { FU }\end{array}$ & $\begin{array}{l}\text { Oxaliplatino } \\
\text { Irinotecan } \\
\text { Cisplatino } \\
\text { Carboplatino } \\
\text { Irinotecan } \\
\text { Topotecan } \\
\text { Gemcitabina }\end{array}$ & $\begin{array}{l}\text { Docetaxel } \\
5 \mathrm{FU}\end{array}$ & Parcial \\
\hline ONCOLC1 & Hepatocelular & 2 & $\begin{array}{l}\text { Sorafenib } \\
\text { Nivolumab }\end{array}$ & $\begin{array}{c}\text { Carboplatino } \\
\text { Oxaliplatino } \\
\text { Irinotecan } \\
5 \mathrm{FU}\end{array}$ & $\begin{array}{c}\text { Cisplatino } \\
\text { Paclitaxel } \\
\text { Docetaxel } \\
\text { Gemcitabina }\end{array}$ & Nula \\
\hline
\end{tabular}


Continúa la Tabla 3: Concordancia entre la progresión a citotóxicos previos y la resistencia a quimioterapia in vitro dependiendo del tumor primario.

\begin{tabular}{|c|c|c|c|c|c|c|}
\hline Identificación & Tumor primario & Línea & $\begin{array}{l}\text { Drogas a las que } \\
\text { progresó }\end{array}$ & Resistencia in vitro & Sensibilidad in vitro & Concordancia \\
\hline AAALC5 & Hepatocelular & 1 & Nivolumab & $\begin{array}{l}\text { Carboplatino } \\
\text { Oxaliplatino } \\
\text { Irinotecan } \\
\text { Gemcitabina }\end{array}$ & $\begin{array}{c}\text { Cisplatino } \\
\text { Paclitaxel } \\
\text { Docetaxel } \\
5 \text { FU }\end{array}$ & Nula \\
\hline AAALC2 & Mama (Luminal B) & 2 & $\begin{array}{l}\text { Palbociclib } \\
\text { Fulvestrant }\end{array}$ & $\begin{array}{l}\text { Carboplatino } \\
\text { Oxaliplatino } \\
\text { Paclitaxel } \\
\text { Irinotecan } \\
5 \text { FU }\end{array}$ & $\begin{array}{c}\text { Cisplatino } \\
\text { Docetaxel } \\
\text { Doxorrubicina } \\
\text { Gemcitabina }\end{array}$ & Nula \\
\hline YDA705 & Mama (Luminal B) & 2 & $\begin{array}{l}\text { Palbociclib } \\
\text { Exemestano }\end{array}$ & $\begin{array}{c}\text { Cisplatino } \\
\text { Paclitaxel } \\
5 \text { FU }\end{array}$ & Doxorrubicina & Nula \\
\hline LUCALC13 & Mama (Luminal A) & 3 & $\begin{array}{l}\text { Palbociclib } \\
\text { Letrozol } \\
\text { Fulvestrant }\end{array}$ & $\begin{array}{l}\text { Carboplatino } \\
\text { Oxaliplatino } \\
\text { Paclitaxel } \\
\text { Irinotecan } \\
\text { Topotecan } \\
5 \text { FU }\end{array}$ & $\begin{array}{l}\text { Cisplatino } \\
\text { Docetaxel }\end{array}$ & Nula \\
\hline AAALC13 & Mama (Luminal B) & 2 & $\begin{array}{l}\text { Paclitaxel } \\
\text { Doxorrubicina } \\
\text { Palbociclib } \\
\text { Letrozol } \\
\text { Goserelina }\end{array}$ & $\begin{array}{l}\text { Paclitaxel } \\
\text { Cisplatino } \\
\text { Carboplatino } \\
\text { Oxaliplatino } \\
\text { Irinotecan } \\
5 \text { FU }\end{array}$ & $\begin{array}{l}\text { Docetaxel } \\
\text { Gemcitabina }\end{array}$ & Parcial \\
\hline AAALC4 & Páncreas & 1 & $\begin{array}{c}5 \mathrm{FU} \\
\text { Oxaliplatino }\end{array}$ & $\begin{array}{c}5 \text { FU } \\
\text { Oxaliplatino } \\
\text { Irinotecan } \\
\text { Gemcitabina }\end{array}$ & & Total \\
\hline YDLCAP8 & Páncreas & 2 & $\begin{array}{c}5 \mathrm{FU} \\
\text { Oxaliplatino } \\
\text { Irinotecan }\end{array}$ & $\begin{array}{c}5 \mathrm{FU} \\
\text { Oxaliplatino } \\
\text { Paclitaxel }\end{array}$ & Gemcitabina & Total \\
\hline LUCALC14 & Páncreas & 3 & $\begin{array}{c}5 \text { FU } \\
\text { Irinotecan } \\
\text { Oxaliplatino } \\
\text { NAB Paclitaxel } \\
\text { Gemcitabina }\end{array}$ & $\begin{array}{c}5 \text { FU } \\
\text { Irinotecan } \\
\text { Oxaliplatino } \\
\text { Paclitaxel } \\
\text { Cisplatino } \\
\text { Carboplatino } \\
\text { Topotecan }\end{array}$ & Docetaxel & Total \\
\hline $\mathrm{ABCLC1}$ & Páncreas & 2 & $\begin{array}{c}\text { Irinotecan } \\
\text { Oxaliplatino } \\
5 \mathrm{FU}\end{array}$ & $\begin{array}{l}\text { Irinotecan } \\
\text { Oxaliplatino } \\
\text { Carboplatino }\end{array}$ & $\begin{array}{c}5 \mathrm{FU} \\
\text { Cisplatino } \\
\text { Paclitaxel } \\
\text { Docetaxel } \\
\text { Gemcitabina }\end{array}$ & Parcial \\
\hline AAALC8 & Páncreas & 1 & $\begin{array}{c}\text { Ninguna (Un ciclo } \\
\text { de FOLFOX) }\end{array}$ & $\begin{array}{c}\text { Cisplatino } \\
\text { Carboplatino } \\
\text { Oxaliplatino } \\
\text { Paclitaxel } \\
\text { Irinotecan } \\
\text { Gemcitabina } \\
5 \text { FU }\end{array}$ & Docetaxel & Nula \\
\hline
\end{tabular}


An Med ABC. 2021; 66 (4): 238-248

Continúa la Tabla 3: Concordancia entre la progresión a citotóxicos previos y la resistencia a quimioterapia in vitro dependiendo del tumor primario.

\begin{tabular}{|c|c|c|c|c|c|c|}
\hline Identificación & Tumor primario & Línea & $\begin{array}{l}\text { Drogas a las que } \\
\text { progresó }\end{array}$ & Resistencia in vitro & Sensibilidad in vitro & Concordancia \\
\hline AAALC6 & Páncreas & 2 & $\begin{array}{l}\text { Cisplatino } \\
\text { Gemcitabina }\end{array}$ & $\begin{array}{l}\text { Cisplatino } \\
\text { Carboplatino } \\
\text { Oxaliplatino } \\
\text { Irinotecan } \\
5 \text { FU }\end{array}$ & $\begin{array}{c}\text { Paclitaxel } \\
\text { Docetaxel } \\
\text { Gemcitabina }\end{array}$ & Parcial \\
\hline
\end{tabular}

Total: las drogas a las que progresó muestran resistencia. Parcial: algunas drogas a las que progresó muestran resistencia. Nula: no hay relación entre la progresión y la resistencia. Con negritas se resaltan aquellos fármacos a los que el paciente progresó previamente y muestran resistencia in vitro.

$5 \mathrm{FU}=5$ Fluorouracilo.

sión clínica, un paciente concordancia parcial y dos pacientes concordancia nula. Un paciente participó en dos ocasiones, antes del inicio del tratamiento y después de tratamiento combinado con 5 Fluorouracilo y Oxaliplatino (AAALC7 y AAALC10). Es interesante observar que la muerte celular con esas dos drogas disminuyó después de la exposición a la quimioterapia.

Se analizaron dos pacientes con carcinoma hepatocelular metastásico a partir de líquido de ascitis, con una y dos líneas de tratamiento previas, ambos mostraron concordancia nula entre la resistencia in vitro y la progresión clínica.

Se analizaron cuatro pacientes con cáncer de mama metastásico, todas de inmunofenotipo luminal, la mediana de líneas de tratamiento en este grupo fue de dos, tres pacientes habían recibido tratamiento hormonal, sólo una había recibido QT. La paciente con quimioterapia mostró concordancia parcial entre la resistencia in vitro y la progresión clínica y las tres pacientes con tratamientos hormonales se catalogaron como concordancia nula por mostrar resistencia a esquemas de quimioterapia.

Se analizaron seis pacientes con cáncer de páncreas metastásico. Los cinco pacientes que habían sido expuestos y progresaron a alguna línea citotóxica mostraron algún tipo de concordancia, tres totales y dos parciales. Un paciente con concordancia nula sin progresión previa.
Se analizó un paciente con cáncer de peritoneo metastásico. Tenía cinco líneas de tratamiento y mostró concordancia total entre la resistencia in vitro y la progresión clínica (Tabla 3).

\section{DISCUSIÓN}

Presentamos los resultados de un ensayo clínico en pacientes con cáncer metastásico y ascitis maligna. A partir del líquido de ascitis se obtuvieron cultivos primarios de células neoplásicas de cada paciente y fueron expuestos a diferentes esquemas de QT para reportar sensibilidad o resistencia de acuerdo con el porcentaje de muerte celular.

El objetivo primario de este estudio fue analizar la concordancia entre la resistencia in vitro y la progresión clínica; sin embargo, otra fortaleza de este estudio es demostrar la factibilidad de realizar estos ensayos en un tiempo razonablemente corto para poder proponerlos como una herramienta de toma de decisiones para el médico oncólogo.

Consideramos que las debilidades de este ensayo clínico son: no poder utilizar fármacos que requieran mecanismos de primer paso y no poder utilizar medicamentos que requiera el sistema inmunológico para su acción, como es el caso de la inmunoterapia.

En cinco muestras no fue posible establecer un cultivo primario de células malignas, probablemente porque se trataba de líquidos de ascitis no maligna. Para responder esta cuestión incluiremos en un fu- 
turo a pacientes con ascitis de etiología no maligna como controles internos.

Un dato discutible es el porcentaje de muerte celular que debemos considerar como punto de corte entre sensibilidad y resistencia. Utilizamos el punto que han propuesto diversos autores. ${ }^{17}$

Sin embargo, en futuros ensayos consideraremos que puede variar de acuerdo con el sitio primario y los esquemas de QT utilizados en el paciente previamente. ${ }^{17-19}$

La lógica inclina a pensar que las células derivadas de pacientes con tumores malignos que aún no son expuestos a ningún tratamiento citotóxico deberían ser sensibles a los esquemas de primera línea; sin embargo, no es así en todos los casos, ${ }^{20,21}$ pues puede existir resistencia a QT intrínseca, independientemente de la exposición previa a los fármacos. También debemos considerar que muchos de los esquemas de tratamiento son combinados, dos drogas que muestran resistencia in vitro por separado podrían mostrar tasa de respuesta objetiva en la clínica cuando se utilicen de forma combinada.

La discordancia entre la resistencia in vitro y la progresión clínica no es necesariamente una debilidad del ensayo clínico o un error en los ensayos. Se puede tratar de pacientes que han progresado a ciertas quimioterapias y continúan mostrando sensibilidad, lo que puede ayudar al clínico a continuar utilizando esta droga en esquemas combinados, como pasa con el 5 Fluorouracilo en cáncer colorrectal. ${ }^{22}$ Otra posibilidad es que se trate de un tumor que es intrínsecamente resistente a ciertos esquemas de QT, dato útil para el médico oncólogo al momento de seleccionar la siguiente línea de tratamiento.

Las células cultivadas muestran rasgos de inmortalidad y malignidad; sin embargo, no sabemos si tienen la capacidad de generar tumores o si estamos seleccionando clonas con este procedimiento. Actualmente en otra línea de investigación de nuestro centro se están inoculando estas células en ratones atímicos en aras de comprobar su tumorigenicidad. Asimismo, en el futuro contemplamos poder ser capaces de determinar la sensibilidad a agentes biológicos e inmunológicos

Si los pacientes muestran tasa de respuesta objetiva después de la exposición a tratamientos que evidenciaron sensibilidad in vitro, será la siguiente fase de este ensayo clínico. El líquido de ascitis no es el único que nos puede aportar células malignas, estamos realizando ensayos similares en líquidos obtenidos a partir de toracocentesis y valoramos expandirlos a líquido cefalorraquídeo y orina.
Presentamos una estrategia innovadora de medicina personalizada con posible uso clínico en los próximos años.

\section{CONCLUSIONES}

Cultivar células neoplásicas a partir de líquido de ascitis en pacientes con cáncer es factible, se puede obtener de procedimientos programados y ofrece una alternativa menos invasiva en comparación con una biopsia. Realizar ensayos de sensibilidad a diferentes esquemas de QT en estos cultivos es factible y se puede hacer en un periodo aproximado de dos semanas.

La concordancia entre la resistencia a quimioterapia in vitro y la progresión clínica fue notoria en los pacientes con cáncer de páncreas y cáncer de peritoneo. Fue parcial en el grupo de cáncer de colon, colangiocarcinoma o mama y fue nula en los pacientes con carcinoma hepatocelular

$\mathrm{Al}$ realizar el ensayo en el mismo paciente en dos puntos diferentes del tratamiento demostramos que los resultados son reproducibles y que el porcentaje de muerte celular disminuye después de que el paciente fue expuesto a cada quimioterapia, lo que sugiere el inicio de mecanismos de resistencia a quimioterapia en los pacientes después de la exposición a quimioterapia.

Este tipo de ensayos podrían servir como una herramienta de medicina personalizada para documentar de forma objetiva y cuantitativa la sensibilidad y resistencia a diferentes quimioterapias en pacientes con cáncer metastásico e incluso, podría en un futuro ser una plataforma de sensibilidad individual en fases más tempranas de la enfermedad.

\section{AGRADECIMIENTOS}

Este trabajo refleja una pequeña fracción de un esfuerzo global. Agradecemos a los residentes y tratantes de oncología médica del CMABC y al Dr. José Luis Ventura Gallegos por la infraestructura para el desarrollo de cultivos celulares.

\section{REFERENCIAS}

1. Chaffer CL, Weinberg RA. A perspective on cancer cell metastasis. Science. 2011; 331 (6024): 1559-1564.

2. Jemal A, Ward EM, Johnson CJ, Cronin KA, Ma J, Ryerson B et al. Annual Report to the Nation on the Status of Cancer, 1975-2014, Featuring Survival. J Natl Cancer Inst. 2017; 109 (9): djx030.

3. National Comprehensive Cancer Network. [Internet]. [Consulted 01 May 2021]. Available in: https://www.nccn.org/ professionals/physician_gls/default.aspx 
4. European Society for Medical Oncology. [Internet]. [Consulted 01 May 2021]. Available in: https://www.esmo.org/Guidelines

5. Sangisetty SL, Miner TJ. Malignant ascites: a review of prognostic factors, pathophysiology and therapeutic measures. World J Gastrointest Surg. 2012; 4 (4): 87-95.

6. Hodge C, Badgwell BD. Palliation of malignant ascites. J Surg Oncol. 2019; 120 (1): 67-73.

7. Liu F, Kong X, Dou Q, Ye J, Xu D, Shang H et al. Evaluation of tumor markers for the differential diagnosis of benign and malignant ascites. Ann Hepatol. 2014; 13 (3): 357-363.

8. Ayantunde AA, Parsons SL. Pattern and prognostic factors in patients with malignant ascites: a retrospective study. Ann Oncol. 2007; 18 (5): 945-949.

9. Sigman M. Introduction: personalized medicine: what is it and what are the challenges? Fertil Steril. 2018; 109 (6): 944-945.

10. Abe K, Wakatsuki T, Katsushima F, Monoe K, Kanno Y, Takahashi A et al. A case of advanced intrahepatic cholangiocarcinoma successfully treated with chemosensitivity test-guided systemic chemotherapy. World J Gastroenterol. 2009; 15 (41): 5228-5231.

11. Dufrenoy J. Les méthodes auxanographiques et leur application au dosage des antibiotiques. Ann Parasitol Hum Comp. 1947; 22 (5-6): 449-479.

12. Shveid D. Caracterización y perfiles moleculares de células tumorales humanas derivadas de cáncer de mama en mujeres con obesidad (Tesis de Maestría). Ciudad de México. Universidad Anáhuac. 2017.

13. Badillo LE. Caracterización biológica de células de cáncer de mama derivadas de pacientes con obesidad. (Tesis de doctorado). Ciudad de México. Universidad Nacional Autónoma de México. 2018.

14. Thériault BL, Portelance L, Mes-Masson AM, Nachtigal MW. Establishment of Primary Cultures from Ovarian Tumor
Tissue and Ascites Fluid, Chapter 24. Malek A. Ovarian Cancer: Methods and Protocols, Methods in Molecular Biology. United States. Springer Science. 2013.

15. Kar R, Chawla D, Gupta B, Mehndiratta M, Wadhwa N, Agarwal R. Establishment of primary cell culture from ascitic fluid and solid tumor obtained from epithelial ovarian carcinoma patients. Int J Gynecol Cancer. 2017; 27 (9): 2000-2005.

16. Li X, Zhu D, Li N, Yang H, Zhao Z, Li M. Characterization of ascites-derived tumor cells from an endometrial cancer patient. Cancer Sci. 2017; 108: 2352-2357.

17. Szulkin A, Otvos R, Hillerdal CO, Celep A, Yousef-Fadhel E, Skribek et al. Characterization and drug sensitivity profiling of primary malignant mesothelioma cells from pleural effusions. BMC Cancer. 2014; 14: 709.

18. Dawson TP, Iyer R V, Lea RW, Roberts P, Harris F, Ashton K et al. The MTS vs. the ATP assay for in vitro chemosensitivity testing of primary glioma tumour culture. Neuropathol Appl Neurobiol. 2010; 36 (6): 564-567.

19. Brigulová K, Cervinka M, Tosner J, Sedláková I. Chemoresistance testing of human ovarian cancer cells and its in vitro model. Toxicol In Vitro. 2010; 24 (8): 2108-2115.

20. Fenizia F, Lambiase M, Aurisicchio L, Normanno N, Ciliberto G, Mancini R. Human lung adenocarcinoma cell cultures derived from malignant pleural effusions as model system to predict patients chemosensitivity. J Transl Med. 2016; 14: 61.

21. Akiyoshia T, Wada T, Nakamurab Y. Clinical correlations with chemosensitivities measured in a simplified tritiated thymidine incorporation assay in patients with malignant effusion. Oncology. 1990; 47: 418-421.

22. Tournigand C, André T, Achille E, Lleldo G, Flesh M, MeryMignard D et al. FOLFIRI followed by FOLFOX6 or the reverse sequence in advanced colorectal cancer: a randomized GERCOR study. J Clin Oncol. 2004; 22 (2): 229-237. 